\title{
A mobilidade urbana em Belo Horizonte/Minas Gerais/Brasil: indicadores e projeções para as viagens por automóveis
}

Daniela Antunes Lessa

Departamento de Geografia, Universidade Federal de Minas Gerais, Belo Horizonte, Brasil.

\author{
Giovanni Candido Miranda \\ Departamento de Cartografia, Universidade Federal de Minas Gerais, Belo Horizonte, Brasil.
}

\section{Carlos Lobo}

Departamento de Geografia, Universidade Federal de Minas Gerais, Belo Horizonte, Brasil.

\section{Leandro Cardoso}

Departamento de Engenharia de Transportes e Geotecnia, Universidade Federal de Minas Gerais Belo Horizonte, Brasil.

Recibido: 8 de febrero de 2018. Aceptado: 25 de octubre de 2018 .

\begin{abstract}
Resumo
O aumento das vendas de automóveis no Brasil nas últimas décadas teve como consequência direta a ampliação do uso do transporte individual em detrimento ao coletivo. As evidências para Belo Horizonte, capital do estado de Minas Gerais, parecem indicar essa tendência, com a gradativa redução do uso do seu principal modo de transporte coletivo: o ônibus. O presente trabalho tem como objetivo o objetivo deste trabalho é analisar a atual magnitude do transporte individual e projetar para 2022 a utilização do automóvel no município de Belo Horizonte/MG/Brasil. A metodologia de projeção utilizou-se das linhas de tendências do modelo de regressão linear e como base de dados a matriz de viagens extraída da Pesquisa Origem e Destino da Região Metropolitana de Belo Horizonte dos anos de 1992, 2002 e 2012, além de um fator de correção baseado na variação da renda domiciliar entre 2000 e 2010. Em geral, as projeções para 2022 indicam a permanência da concentração das viagens por automóvel na área central, pericentral e nas regiões de maior renda do município, além do expressivo crescimento desse modo nas periferias. Há, nesse sentido, a iminente necessidade de melhorias no transporte público frente à realidade que se apresenta para os próximos anos.
\end{abstract}

Palavras-chave

Mobilidade urbana Transporte individual Transporte coletivo Projeções de fluxos Pesquisa origem destino 


\section{Urban mobility in Belo Horizonte/Minas Gerais/Brazil: indicators and projections for travel by automobiles}

\begin{abstract}
The increase in car sales in Brazil in the last decades has had the direct consequence of expanding the use of individual transportation to the detriment of the collective. Evidence for Belo Horizonte, capital of the state of Minas Gerais, seems to indicate this tendency, with the gradual reduction of the use of its main mode of collective transportation: the bus. The present work aims to analyze the current conditions and to design the use of transportation by car in the displacements developed in Belo Horizonte. The projection methodology used the trend lines of the linear regression model and as data base the trip matrix extracted from the Pesquisa Origem e Destino da Região Metropolitana de Belo Horizonte from the years of 1992, 2002 and 2012, as well as a factor based on the variation of household income between 2000 and 2010 . In general, the projections for 2022 indicate the permanence of the concentration of travel by car in the central, pericentral and higher income regions of the municipality, in addition to the expressive growth of this mode in the peripheries. There is, in this sense, the imminent need for improvements in public transportation facing the reality that presents itself for the next years.
\end{abstract}

\section{Introdução}

O sistema de transporte tem como objetivo central garantir a mobilidade, o acesso e a integração espacial dos bens e indivíduos às diferentes atividades, que são definidas e condicionadas pelo uso e ocupação do solo. Além disso, o sistema de transporte influencia diretamente os custos de produção, os fluxos de comércio, a determinação de áreas de mercado para as regiões e o bem-estar social da população, tendo assim um papel importante no crescimento e desenvolvimento econômico das cidades (Mitra e Saphores, 2016). Portanto, o sistema de transporte urbano possui uma estreita relação com a forma e a estrutura espacial (Rodrigue et al., 2013).

O modelo de produção e organização do espaço predominante nas grandes cidades do Brasil tem como característica marcante o alto grau de periferização e dispersão locacional das atividades econômicas. Essa condição resulta na exigência de grandes deslocamentos da população para atividades relacionadas ao trabalho, estudo e lazer. De maneira semelhante às principais cidades dos países periféricos, no Brasil existe grande dependência dos deslocamentos a pé e dos meios de transporte público o deslocamento de pessoas e mercadorias. Embora o sistema coletivo por ônibus seja o modo de transporte mais utilizado nos países periféricos (Vasconcellos, 2000), observa-se no Brasil um crescimento expressivo do uso do transporte individual motorizado nos últimos anos. A concessão de maior volume de subsídios em detrimento do transporte público (Vasconcellos et al., 2011), aliada ao aumento da renda e do crédito na primeira década do século XXI, provocou o aumento do número de veículos particulares nas cidades brasileiras, em especial aqueles realizados por automóveis e/ou motocicletas.

Belo Horizonte, capital do estado de Minas Gerais, de acordo com o Instituto Brasileiro de Geografia e Estatística (IBGE, 2017), é atualmente a sexta cidade mais populosa do Brasil, com uma população residente estimada de 2.523.794 habitantes em 2017. Sua região metropolitana é a terceira do país em tamanho demográfico, com um total de 5.915.536 habitantes. De maneira similar ao que ocorre nas demais metrópoles brasileiras, Belo Horizonte apresenta uma série de problemas relacionados ao acelerado e excludente

\section{Keywords}

Urban mobility Individual transportation Public transportation Flows projection Origin destination research

\section{Palabras clave}

Movilidad urbana Transporte individual Transporte colectivo Proyección Encuesta origen-destino 
processo de urbanização, que, aliados à gestão ineficiente e desarticulada do sistema de transporte público, resultou em um descompasso entre a demanda de qualidade e a capacidade do Estado em suprir a necessidade de mobilidade de maneira adequada e justa. A crescente utilização dos modos individuais de transporte motorizado, principalmente dos automóveis, é um dos grandes desafios da política de mobilidade no município, exigindo esforços do poder público ações efetivas que proporcionem um melhor ir e vir.

Nesse contexto, o objetivo deste trabalho é analisar a atual magnitude do transporte individual e projetar para 2022 a utilização do automóvel no município de Belo Horizonte/MG/ Brasil. Para tanto, foram utilizadas as bases de dados da Pesquisa Origem e Destino (OD) dos anos de 1992, 2002 e 2012 e dos censos demográficos do IBGE de 2000 e 2010. Os indicadores apresentados, bem como as projeções dos fluxos de origem, permitiram avaliar o peso dos deslocamentos por automóvel em cada uma das unidades espaciais denominadas Campos. Os resultados obtidos no trabalho permitem, apesar os limites inerentes ao recorte temporal analisado, permitem identificar áreas no município com maior necessidade de intervenção, seja por medidas que visam reduzir as restrições e ampliar a eficiência do sistema, ou por investimento e estimulo ao na utilização do transporte coletivo.

\section{Cidade e mobilidade: desafios ao sistema de transporte público}

A urbanização se apresenta como um dos processos dominantes na contemporaneidade. De acordo com relatório das Nações Unidas, no ano de 2014 cerca de 54\% da população mundial habitava áreas urbanas. Se em 1950 cerca de 30\% da população mundial estava no espaço urbano, as projeções da própria ONU indicam que essa proporção atingirá 66\% em 2050 (United Nations, 2014). Essa mudança não é apenas um dado isolado, mas representa mudanças profundas na organização social, que envolve a prevalência de novos modos de vida. Como bem descreve Lefebvre (1969), a cidade representa a mediação de relações entre processos globais (econômicos, sociais políticos e culturais) e ritmos locais que modificam o espaço urbano. É na cidade contemporânea que os anseios de mobilidade social e espacial dos indivíduos e grupos são traduzidos (Berman, 2007). De acordo com Vasconcellos (2001), é a circulação (mobilidade urbana) que liga fisicamente todas as atividades no espaço. A ligação entre os diferentes espaços, responsável pela mediação entre estruturas de produção e a vida social em geral, é realizada pelas estruturas construídas para permitir a circulação no espaço urbano (Dear e Scott, 1981).

Para Cheng e Chen (2015) a mobilidade pode ser definida como a capacidade de se deslocar de um ponto ao outro. Jones (1981) e Cardoso (2007) definem a mobilidade como a capacidade de um indivíduo acessar determinada localidade, o que envolve o serviço de transporte e sua condição física e financeira. Gutiérrez (2012) complementa o conceito mobilidade incluindo a ideia de materialização de viagens motivadas por necessidades e/ou desejos de realizar atividades, uma vez que algumas dessas necessidades podem ser atendidas por serviços de telecomunicações. Há, portanto, uma estreita relação entre estratégias e políticas de transporte, que compreende um círculo "vicioso" envolvendo o uso do solo, o intercâmbio de atividades, o transporte, a acessibilidade e a mobilidade (Rodrigue et al., 2013). Acessibilidade, desde o século passado, tem sido utilizada em diferentes vertentes do conhecimento científico, figurando com destaque nas áreas de planejamento urbano e de transportes (Vulevic, 2016); na influência do uso do solo (Harris, 1954); como agente e medida de segregação socioespacial (Pyrialakou et al., 2016); e como indicador de acesso de pedestres à infraestrutura de circulação (Ewing e Handy, 2009). A acessibilidade envolve, portanto, a combinação da localização dos destinos a serem alcançados e as características do sistema de transporte, considerando a distribuição geográfica da população e das atividades econômicas, assim como as respectivas características (Tagore e Sikdar, 1995). 
Por sua vez, a mobilidade não se refere apenas ao melhor deslocamento de um ponto a outro do território (circulação), ou à existência de infraestrutura viária e de diversos modos de transporte, combinada à localização dos destinos a serem alcançados (acessibilidade), o seu conceito incorpora a circulação e a acessibilidade, e "trata de outro movimento, justamente o que permite ampliar as possibilidades de apropriação e uso coletivo e individual do espaço urbano" (Barbosa, 2014:198). Gomide (2006) também considera a facilidade de deslocamento das pessoas e bens na cidade, tendo em vista a complexidade das atividades econômicas e sociais nela envolvidas. Essa facilidade, como demonstrou Vasconcellos (2001), envolve dois componentes: (I) desempenho do sistema de transporte, por sua capacidade de interligar locais distintos; e (II) características próprias do indivíduo, relacionadas às suas condições física e econômica, ao seu grau de inserção e necessidades do sistema de transporte.

Maggi e Vallino (2016) enumeram alguns fatores que influenciam a mobilidade, como, por exemplo, as dimensões físicas do espaço urbano, as múltiplas atividades desenvolvidas, a disponibilidade de serviços de transporte, bem como as características da população (renda, faixa etária e escolaridade). Assim, "a mobilidade urbana mostra-se como a necessidade por transporte, moldada pelo modo de vida da população" (Raia Junior, 2000), e pode ser considerada como um importante recurso social e articulador na sociedade, uma vez que está diretamente relacionada ao movimento de pessoas entre diferentes hierarquias sócio espaciais (Costa et al., 2017). Barbosa (2014) também afirma que a mobilidade não se refere apenas ao tempo de duração de viagens ou a eficácia do sistema viário para deslocar pessoas. Ela não significa simplesmente um deslocamento eficiente entre pontos do território para o exercício de atividades econômicas, sociais e culturais. Esse significado, comumente atribuído à mobilidade, se remete na realidade à circulação intra ou entre lugares. De acordo com esse autor, a mobilidade inclui a circulação, mas a supera, pois trata de um movimento que permite ampliar as possibilidades de apropriação e uso coletivo e individual do espaço urbano. Assim, o estudo da mobilidade envolve os deslocamentos efetivos de bens e pessoas e os seus padrões, que resultam nas condições de acesso dos diferentes grupos sociais à vida cotidiana (Gutiérrez, 2012).

Os problemas relacionados à mobilidade urbana aumentaram com a urbanização. De maneira geral, são reflexos diretos do acelerado processo de expansão e crescimento do tecido urbano, que resulta na concentração espacial da população e das demandas de mobilidade em áreas específicas (Rodrigue et al., 2013). Litman (2017) afirma que as questões que envolvem o planejamento e a gestão do espaço urbano sempre estiveram relacionadas ao sistema de transportes. Existe, portanto, uma interdependência entre crescimento urbano e a demanda e oferta de transportes, de maneira que deficiências no planejamento urbano e de transportes interferem diretamente no cotidiano da população. O crescimento urbano acelerado; a dispersão espacial; o aumento no número de automóveis nos centros urbanos, de congestionamentos e de acidentes de trânsito; as deficiências no transporte coletivo; e as questões de uso do solo e de ordenamento espacial são reflexos da dissociação entre os aspectos do planejamento urbano e de transporte, e têm levado a uma deterioração da qualidade de vida da população nos centros urbanos (Schoenau e Müller, 2017).

\section{Transporte público e privado nas metrópoles brasileiras: evidências e contradições}

De acordo com o Censo Demográfico do Instituto Brasileiro de Geografia e Estatística, o Brasil atingiu em 2010 o percentual de $84,34 \%$ de sua população residente em áreas urbanas. Esse fato tem representado sensíveis mudanças no padrão de mobilidade de sua população, caracterizado pela manutenção e ampliação de elevados custos sociais, 
econômicos e ambientais, reflexo, principalmente, do uso cada vez mais acentuado do transporte motorizado individual (Carvalho, 2016). Para Neto (2004) a produção de transporte urbano no Brasil se dá com altos níveis de ineficiência e irracionalidade, com a transformação das maiores cidades brasileiras em espaços eficientes para o automóvel. Ao mesmo tempo em que o uso do transporte individual cresce desde os anos 1990, a demanda por transporte público sofre quedas frequentes. Como demonstrado por Araújo et al. (2011), a frota de automóveis no país cresceu substancialmente nas últimas décadas, apresentada como a única alternativa eficiente de transporte para as populações com níveis mais altos de renda.

A utilização em larga escala do automóvel modificou a distribuição modal do transporte urbano, ocasionando a deterioração dos transportes coletivos (Gomide, 2006). As políticas públicas no Brasil têm contribuído para o aumento da motorização da população, algo comprovado por maiores subsídios ao transporte individual em detrimento do transporte público. Vasconcellos et al. (2011) aponta que do total de subsídios do Estado voltados ao transporte urbano em $2005,90 \%$ se direcionaram aos automóveis. Nesse cálculo, os autores incluem os subsídios relacionados à aquisição de automóveis, como a redução do imposto sobre produtos industrializados (IPI), a não cobrança de estacionamento nas vias públicas, os valores do imposto sobre a propriedade de veículos automotores (IPVA) e licenciamento de veículos, além dos subsídios concedidos para os táxis. Por sua vez, o subsídio ao transporte público ocorre principalmente na aquisição de veículos e sua operação (redução de impostos), não existindo subsídio direto às passagens. A tendência observada diante do aumento do uso do automóvel é a perda da competitividade do transporte público rodoviário, ocasionado pelo estímulo ao transporte privado, aumento dos custos e consequente perda de demanda, gerando aumentos das tarifas e retroalimentando um círculo vicioso (Vasconcellos et al., 2011).

O automóvel recebeu o errôneo status de solução para os problemas de mobilidade nos diferentes extratos de renda da sociedade brasileira. De acordo com Zandonade e Moretti (2012), a parcela de maior renda da população se utiliza exclusivamente do automóvel, comportamento que tende a ser seguido pelos setores de renda média. Por sua vez, parte da população de menor renda, por ter condições de transporte precárias, compromete importante parcela do orçamento na tentativa de aquisição do automóvel, com o objetivo de sanar os problemas de mobilidade em áreas precárias da cidade. $\mathrm{O}$ acesso ao automóvel se ampliou na primeira década do século XXI, com a redução dos preços dos automóveis (devido às isenções fiscais às montadoras) e facilidades de financiamento no Brasil. Portanto, uma tendência onerosa se caracteriza: com mais automóveis nas ruas, há maior demora dos ônibus devido aos congestionamentos, resultando em maior tempo de viagem, maior lotação e maiores custos.

$\mathrm{O}$ atual modelo de mobilidade urbana brasileiro amplia os custos ambientais, sociais e econômicos a toda sociedade. Porém, esses custos são divididos de maneira desigual entre a população. Quem mais sofre com o aumento de automóveis nas ruas é a parcela da sociedade que não utiliza o automóvel como meio de locomoção (Zandonade e Moretti, 2012). Dessa forma, a crise da mobilidade urbana no Brasil afeta em maior grau as camadas mais pobres da sociedade, subordinadas a sistemas de locomoção inadequados, pelos quais devem pagar uma parcela cada vez maior dos seus ganhos (Cardoso, 2007). Carvalho (2016) ainda destaca que os gastos com transporte público são crescentes apenas para famílias mais pobres, em função do aumento dos deslocamentos. Porém, mesmo nessa faixa de renda, os gastos com transporte privado são maiores. Para a população de maior poder aquisitivo, os gastos per capita com transporte público decrescem. Verifica-se, contudo, que as famílias brasileiras apresentam uma característica de gastar menos em transporte público e mais em transporte privado em praticamente todas as faixas de renda. Contudo, esses gastos com transporte privado sobem exponencialmente à medida que a renda aumenta (Carvalho, 2016). 
O aumento da renda resulta na maior possibilidade de deslocamentos dos indivíduos, o que resulta em uma maior diversidade de atividades realizadas (Vasconcellos, 2001). Quando são consideradas apenas as viagens motorizadas, a variação da mobilidade é ainda maior. As distâncias percorridas aumentam de acordo com o poder aquisitivo, uma vez que as pessoas de renda mais alta dispõem de modos de transporte mais rápidos e percorrem maiores distâncias no mesmo tempo disponível. O espaço de circulação é "consumido" (acessado) de forma diferenciada, refletindo assim diferentes condições sociais e econômicas. Dessa forma, as condições de mobilidade afetam diretamente o desenvolvimento econômico das cidades, a interação social e o bem-estar de seus habitantes (Lobo et al., 2013). Esse cenário tem contribuído para a deterioração das condições de mobilidade da população dos grandes centros urbanos, materializada no crescimento dos acidentes de trânsito com vítimas, dos congestionamentos urbanos e também dos poluentes veiculares (Costa et al., 2017).

De acordo com Carvalho (2016), a percepção geral é que essas condições continuarão por muito tempo, pois as políticas de incentivo à produção, venda e utilização de veículos privados prevalecem sobre as medidas de estímulo ao uso do transporte público e do transporte não motorizado. As tendências de aumento do transporte individual no Brasil ainda são fortes, trazendo, dessa forma, grandes desafios para os dirigentes e gestores públicos do transporte no que tange ao planejamento de políticas mitigadoras das externalidades negativas produzidas. Novas abordagens têm sido introduzidas na gestão e no planejamento urbano, associando o transporte público, a circulação e o planejamento das atividades urbanas no que hoje se denomina como planejamento da mobilidade (Oliveira e Silva, 2015). Novas ferramentas vêm sendo desenvolvidas para avaliar a mobilidade urbana, entre elas, os índices e indicadores, que podem ser aplicados nas análises de impactos econômicos, sociais, ambientais ou em aspectos específicos de sustentabilidade, tais como acessibilidade, mobilidade e capacidade ambiental (Nicolas et al., 2003). Essa é uma das vertentes que esse trabalho investe.

\section{A mobilidade por automóvel em Belo Horizonte: uma breve contextualização histórica}

Belo Horizonte, capital do estado de Minas Gerais, foi uma das primeiras cidades brasileiras planejadas desde sua concepção. O projeto elaborado no final do século XIX pelo engenheiro Aarão Reis buscava referências de ideologias positivistas e modernistas, inspirado nas cidades de Paris e Washington, ao trazer uma arquitetura marcada por ruas e avenidas largas e retas em sua zona urbana, delimitada, à época, pela Avenida do Contorno (Jayme e Trevisan, 2012). Ao longo do século XX a cidade passou por importantes transformações, impulsionadas, principalmente, pelo grande crescimento populacional registrado no período.

Na década de 1920, a Avenida do Contorno deixava de ser o limite da zona urbana da cidade para delimitar apenas sua área central. Entre os anos 1930 e 1950, [...] iniciava-se um processo de verticalização da área central. [...] A década de 1960 foi marcada por [...] interesses do capital e do automóvel. As ruas do centro, [...] foram tomadas pelos carros, se tornando cada vez mais lugares de passagem (Jayme e Trevisan, 2012:362).

A presença do automóvel no cenário urbano de Belo Horizonte se intensificou ao longo da década de 1970. Destaca-se, ainda, que a população residente em Belo Horizonte passou de 1.235.030 em 1970, para 2.375.151 em 2010, segundo dados dos censos demográficos brasileiros. Paralelamente, de acordo os relatórios do Departamento Nacional de Trânsito (DENATRAN), observou-se uma considerável expansão da frota de veículos automotores na capital mineira, que acompanhou o cenário mundial e alcançou a marca de $1.340 .071 \mathrm{em} 2010$. Apenas entre os anos de 2000 e 2010, enquanto a taxa de crescimento da população foi de $6,10 \%$, a frota de veículos registrou um espantoso 
incremento de $97 \%$ (SISMOB-BH, 2015). Houve um crescimento exponencial da frota registrado ao longo da década de 2000, entre os anos de 2005 e 2010 o índice registrado foi de 70\%. Nesse mesmo período, de acordo com relatórios do DENATRAN, a quantidade de automóveis nas ruas de Belo Horizonte aumentou em 51\%.

O crescimento dos modos individuais no cenário urbano da Capital foi confirmado pelos dados da Pesquisa OD de 2012. Pela primeira vez, desde o ano de 1972, quando se iniciam os estudos dos padrões de deslocamento da população belo-horizontina, foi registrada uma inversão na divisão modal. Nesse levantamento se pode observar que a maior parcela dos deslocamentos diários, realizada até então pelos modos coletivos, passou a ser feita pelos modos individuais motorizados. No ano de 2016, a taxa de motorização registrada em Belo Horizonte foi de 0,70 veículo por habitante. Em 2000 essa taxa era de 0,56 . No cenário urbano, a tendência crescente da taxa de motorização potencializa esse problema. O individualismo colabora significativamente para o aumento dos congestionamentos que, em geral, são causados pelo excesso de veículos em horários específicos e por acidentes ou ocorrências, como problemas mecânicos, condições do tempo, obras e reparos, entre outras, que podem bloquear as pistas (Resende e Souza, 2009). Embora a taxa de motorização de Belo Horizonte seja próxima da média de países desenvolvidos, existem características ainda mais desfavoráveis que fazem o trânsito do município ser vivenciado de maneira mais intensa, como o planejamento urbano ineficaz, o uso exagerado do veículo particular, além das más condições do transporte público, de forma a não permitir que o crescimento da motorização no município seja acompanhado de avanços no bem-estar urbano (Lobo et al., 2013).

\section{Base de dados, unidade espacial de analise e procedimentos metodológicos}

As Pesquisas Origem e Destino (ODs) são consideradas a mais completa base de dados sobre a demanda por transporte humano. Registram os movimentos realizados pelos indivíduos por meio de entrevistas domiciliares nas principais Regiões Metropolitanas do Brasil. Essas pesquisas apresentam informações sobre o deslocamento realizado (motivo, horário, modo, origem, destino e tempo de viagem) e sobre as pessoas envolvidas (idade, escolaridade e renda). No Brasil, a primeira Pesquisa OD foi aplicada em São Paulo no ano de 1967 (ANTP, 1997). A OD da Região Metropolitana de Belo Horizonte foi realizada pela primeira vez em 1972, sendo repetida nos anos de 1982, 1992, 2002 e 2012. Nessa pesquisa, todos os ocupantes do domicílio objeto de estudo foram questionados a respeito do número de deslocamentos realizados no dia anterior, o modo de transporte, o motivo, a duração, horário e destino das viagens.

Para definir a configuração espacial da Região Metropolitana de Belo Horizonte (RMBH), foi implementado um sistema de unidades espaciais, para assim analisar o movimento desses grupos e suas relações com transformações sócio espaciais. Esse sistema foi consolidado por equipes da Fundação João Pinheiro (FJP) de forma a possibilitar a análise de séries históricas. A menor unidade espacial de coleta é a Área Homogênea (AH), que engloba um conjunto de setores censitários agregados, definidos pelos Censos Demográficos, de conforme critérios socioeconômicos e de ocupação territorial. As AHs podem ser agregadas em Campos, subcomplexos diferenciados de Campos, complexos diferenciados de Campos e macrounidades (Minas Gerais, 2002). O primeiro nível de agregação das AHs, de acordo com o sistema de unidades espaciais da OD/RMBH, é denominado Campo. No aglomerado metropolitano, o Campo é considerado o nível que melhor retrata o cotidiano e seu conceito se aproxima do que constituiria um bairro ideal, cuja identidade de seus moradores é mantida (Minas Gerais, 2012). 
As bases de dados utilizadas compreendem as ODs de 1992, 2002 e 2012, desenvolvidas, respectivamente, pelo antigo Núcleo de Ensino e Pesquisa em Transporte (NEPT) do Departamento de Engenharia de Transportes e Geotecnia (ETG) da Universidade Federal de Minas Gerais (UFMG); pela Fundação João Pinheiro (FJP); e pela Agência de Desenvolvimento da Região Metropolitana de Belo Horizonte, órgão vinculado ao Governo do Estado de Minas Gerais. Foi utilizada a compatibilização espacial adotada pela Fundação João Pinheiro (FJP) entre os anos de 1992 e 2002, e pela Empresa de Transporte e Trânsito de Belo Horizonte (BHTRANS) entre as ODs de 2002 e 2012, estabelecendo um total de 106 Campos no município de Belo Horizonte. A Figura 1 representa os respectivos Campos compatibilizados, distribuídos conforme as nove regionais administrativas de Belo Horizonte (Barreiro, Centro-Sul, Leste, Nordeste, Noroeste, Norte, Oeste, Pampulha e Venda Nova).
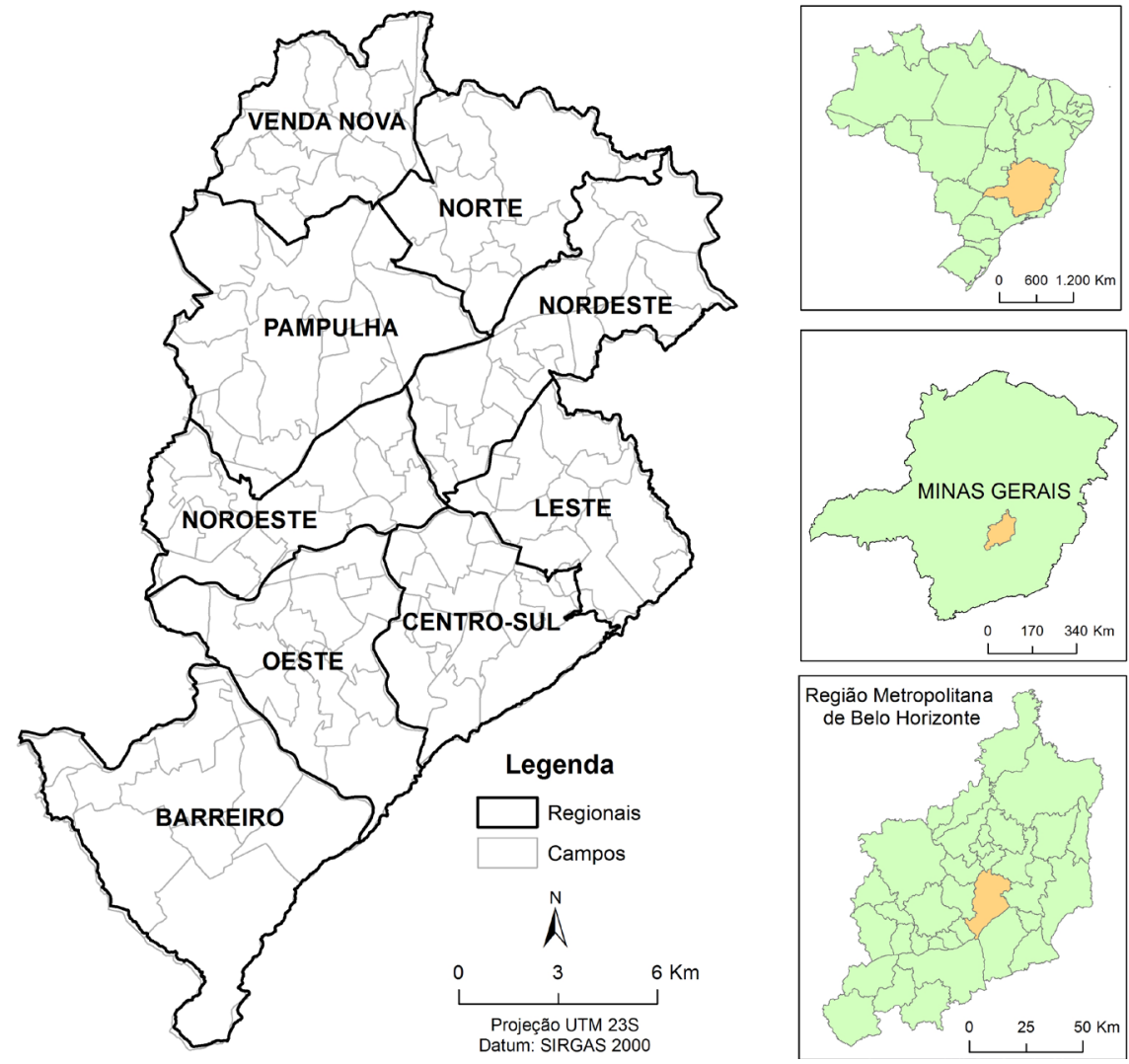

Figura 1. Campos por regionais administrativas de Belo Horizonte. Fonte: Elaboração própria a partir da base digital das Pesquisas OD (1992, 2002 e 2012) - FJP.

A elaboração das projeções se utiliza dos princípios da análise de regressão, tendo como base a linha de tendência linear, e a equação que tenta explicar alterações na variável dependente $(\mathrm{Y})$ pela variação dos níveis $\mathrm{da}(\mathrm{s})$ variável(is) independente(s) (X). O estabelecimento do modelo para a explicação do fenômeno deve verificar qual tipo de curva e equação de um modelo matemático mais se aproxima dos pontos representados no diagrama de dispersão. Haverá, porém, na maior parte dos pontos, uma distância entre os pontos do diagrama e a curva do modelo matemático, já que os pontos são influenciados por fatores externos não identificados no modelo. Dessa forma, o propósito da regressão é obter um modelo matemático que melhor se ajuste aos valores observados de $\mathrm{Y}$, em função da variação dos níveis da variável $\mathrm{X}$. O modelo estatístico para a regressão linear simples é dado pela Equação 1 . 


$$
Y=\alpha X+\beta+\varepsilon
$$

em que Y: Valor observado (variável dependente); $\alpha$ : Coeficiente angular (inclinação da reta; X: Variável explicativa (variável independente); $\beta$ : Intercepto; e $\varepsilon$ : Componente aleatória (erro).

O modelo se utiliza de uma regressão linear, em que a variável dependente (Y) é expressa pelo número de viagens por automóveis, respectivamente, originadas em cada Campo e a variável independente (X) é expressa pelo tempo (1992, 2002 e 2012). Com base nos dados de viagens e no tempo foi definida uma equação da reta com tendência linear para cada Campo e modo estudado. O modelo linear se baseia na hipótese de que as causas da variação nos anos anteriores devem se manter constantes no tempo. O modelo de projeção utilizado foi adaptado ao método AiBi - denominado originalmente "Apportionment Method" (WALDVOGEL, 1998). O propósito é projetar assumindo a tendência linear dos valores em um período pré-determinado.

Os testes do modelo de regressão linear utilizado foram estatisticamente consistentes quando comparados os resultados da projeção para 2012 com os valores observados para esse mesmo ano (teste de consistência do modelo). Os coeficientes de corelação de Pearson, que medem a força e a direção da relação linear entre as variáveis quantitativas, foram 0,999 e 0,997, respectivamente. As análises de variância apresentaram altos F-statistics e baixas significâncias, indicando variação baixa dos resíduos, revelando a adequabilidade dos modelos. Os valores de p-value também se mostraram dentro do intervalo de confiança de 95\%, sendo rejeitada as hipóteses nulas, o que atestou uma significativa correlação entre as variáveis em análise.

Além do modelo de regressão, também foi utilizado um fator de ajuste, calculado com base no crescimento da renda média do responsável pelo domicílio em $\mathrm{R} \$$ nos anos de 2000 e 2010. Embora o valor da moeda nacional possa ter sofrido depreciação quanto ao valor de compra, a função derivada avalia apenas o nível diferenciado de crescimento, ajustando a projeção linear positivamente daqueles que tiveram crescimento mais elevado na renda.. O fator de ajuste (fa), descrito na Equação 2, foi obtido pela razão entre a taxa geométrica de crescimento médio da renda padronizada (Equação 4) e proporção da taxa de crescimento total das viagens em cada Campo.

$$
f a(x)=\operatorname{tgcm}_{p}+\left(\frac{v_{i}}{\sum_{n=1}^{\infty} v}\right)
$$

em que, v_i: total de viagens no n-ésimo Campo; v: total de viagens em Belo Horizonte e tgcm: taxa geométrica de crescimento médio da renda, dada pela Equação 3.

$$
\operatorname{tgcm}=\left(\left(\frac{v_{i_{2010}}}{v_{i_{2000}}}\right)^{\frac{1}{n}}\right)-1
$$




$$
\operatorname{tgcm}_{p}=\frac{x_{i}-\mu}{\sigma}
$$

em que, $\mathrm{n}$ : número de anos; $\mathrm{x} \_\mathrm{i}$ : taxa de crescimento da renda no $\mathrm{n}$-ésimo Campo; $\mu$ : média do crescimento da renda no município; e $\sigma$ : desvio padrão do crescimento da renda no município.

Ao final, o valor final corrigido foi dado pela taxa de crescimento projetada somada ao fa. Embora os valores possam apresentar variações decorrentes da alteração no valor de compra de um período para o outro, o fator funciona apenas na redistribuição do crescimento esperado, tendo como base as alterações na renda local no período observado (2000/2010).

\section{As viagens por automóveis, do observado ao esperado: análise e interpretação dos resultados}

Com base nos dados das pesquisas OD, verifica-se o aumento do número de viagens realizadas dentro do limite territorial da Região Metropolitana de Belo Horizonte. No ano de 1992, foram registradas cerca de 3,8 milhões de viagens. Entre os anos de 2002 e 2012 foi registrado um aumento de 3,9 para 6,8 milhões de viagens. No entanto, esse crescimento não se deu de maneira semelhante nos diferentes modos. Em geral, nota-se um incremento mais expressivo nas viagens por automóveis (Figura 2). No ano de 1992, foram realizadas 460.169 viagens por automóvel, que representavam 12,2\% do total e passou, em 2012, para cerca de 1,5 milhões, que representavam $21,5 \%$. No que tange as viagens por ônibus, esse número passou de cerca de 1,8 milhões (46,5\%) para 1,6 milhões (23,3\%).

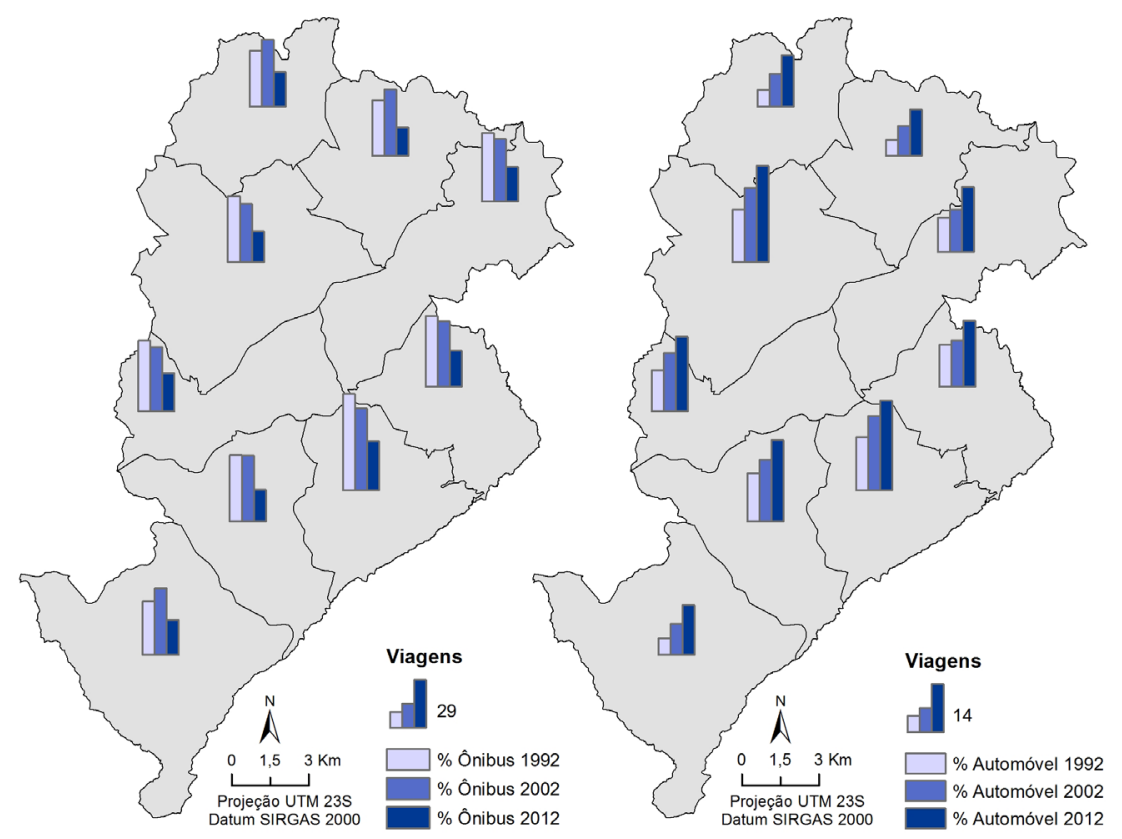

Figura 2. Participação dos modos de transporte no total de viagens realizadas com origem em Belo Horizonte por regional administrativa. Fonte: Elaboração própria a partir da base digital das Pesquisas OD (1992, 2002 e 2012). 
O crescimento do número de viagens por automóvel (Figura 3) ocorreu em todas as regiões de Belo Horizonte, incluindo as de menor renda. Entre 1992 e 2002 esse aumento foi de $41,9 \%$. Por sua vez, entre 2002 e 2012 o número de viagens realizadas por automóvel (excluindo-se as viagens em que o indivíduo entrevistado era passageiro do veículo) aumentou em $124 \%$. A consequência do crescimento no número de viagens por automóvel em Belo Horizonte foi o aumento do tempo gasto nos deslocamentos (Lobo et al., 2012). A errônea interpretação dada pelos indivíduos de que o transporte individual motorizado é a única solução para os problemas do trânsito no município acaba por gerar um maior uso do automóvel e, também, de outros meios de transporte como as motocicletas. É importante salientar a política de redução de impostos sobre produtos industrializados (IPI) na primeira década do século XXI desenvolvida pelo governo federal. Tal decisão, aliada à ampliação da renda (principalmente por meio do aumento do salário mínimo) e do crédito impulsionou a venda de automóveis e motocicletas no Brasil entre 2002 e 2012. De acordo com dados da Confederação Nacional dos Transportes (CNT), em 2015, Belo Horizonte apresentou a maior taxa de motorização dentre as 17 capitais brasileiras, com um índice de 0,65 veículo por habitante.

Uma comparação detalhada entre o uso do ônibus e do automóvel em Belo Horizonte pode ser efetuada com o uso do indicador denominado Razão de Mobilidade por Automóvel. Esse indicador demonstra o número de viagens por automóvel para cada viagem por ônibus realizada no referido Campo. Índices maiores que 1 indicam a prevalência do uso do transporte por automóvel, enquanto os valores abaixo de 1 demonstram o predomínio do transporte público por ônibus. A Figura 4, que representa a Razão de Mobilidade por Automóvel em Belo Horizonte, permite observar que, em 1992, as viagens por automóvel prevaleciam em quatro Campos (que corresponde a 3,66\% do total). Essa situação se alterou em 2012, com um expressivo crescimento do uso do automóvel nos deslocamentos diários. Um total de 49 Campos (44,95\%) registrou maior número de viagens por esse modo de transporte individual, se comparado ao ônibus. Nota-se, portanto, que, embora a predominância dos deslocamentos por ônibus nos Campos tenha decaído entre os anos de 1992 e 2012, o modo coletivo ainda sobreleva o individual.

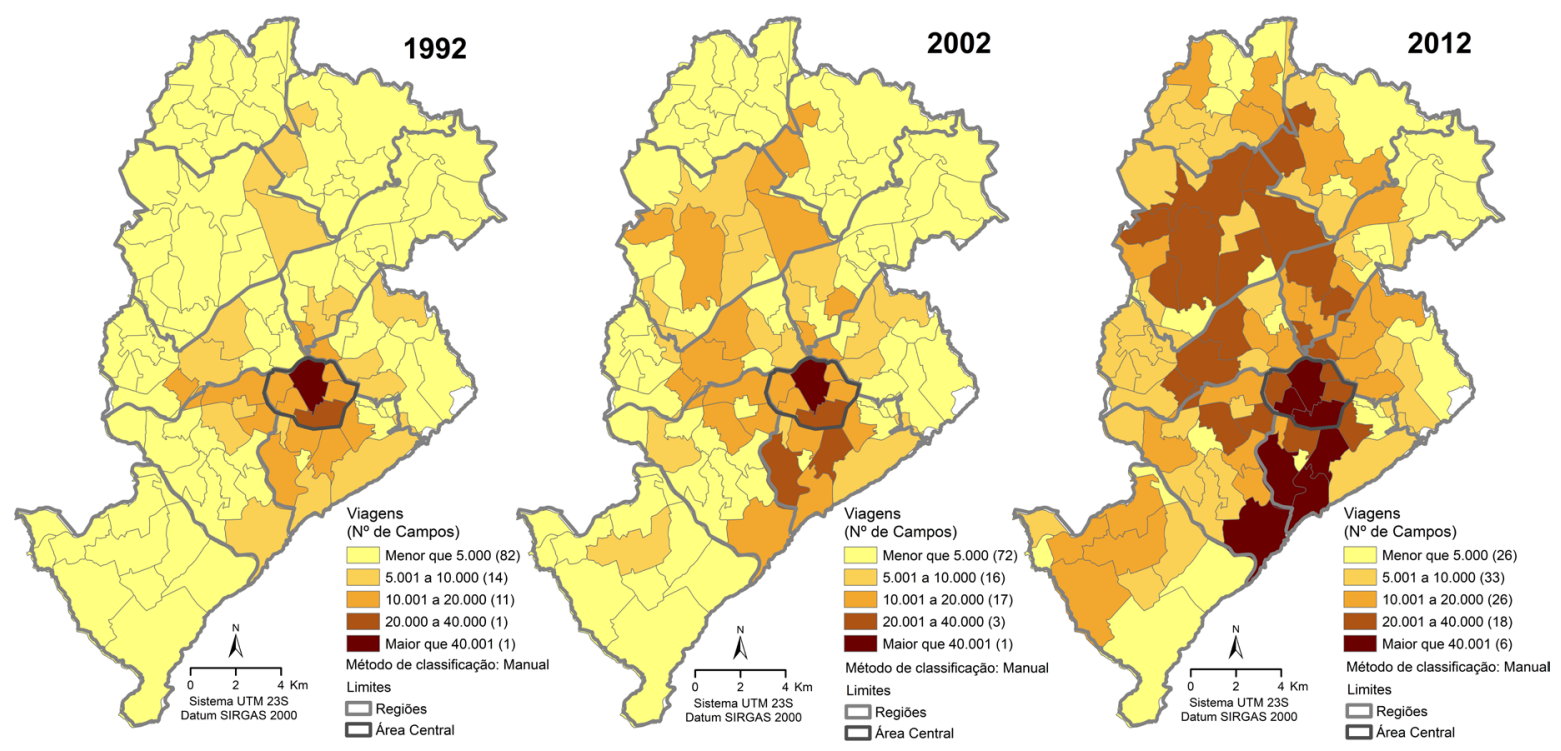

Figura 3. Total de viagens por automóveis com origem em Belo Horizonte por Campos, 1992, 2002 e 2012. Fonte dos dados: Elaboração própria a partir da base digital das Pesquisas OD (1992, 2002 e 2012) - FJP. 


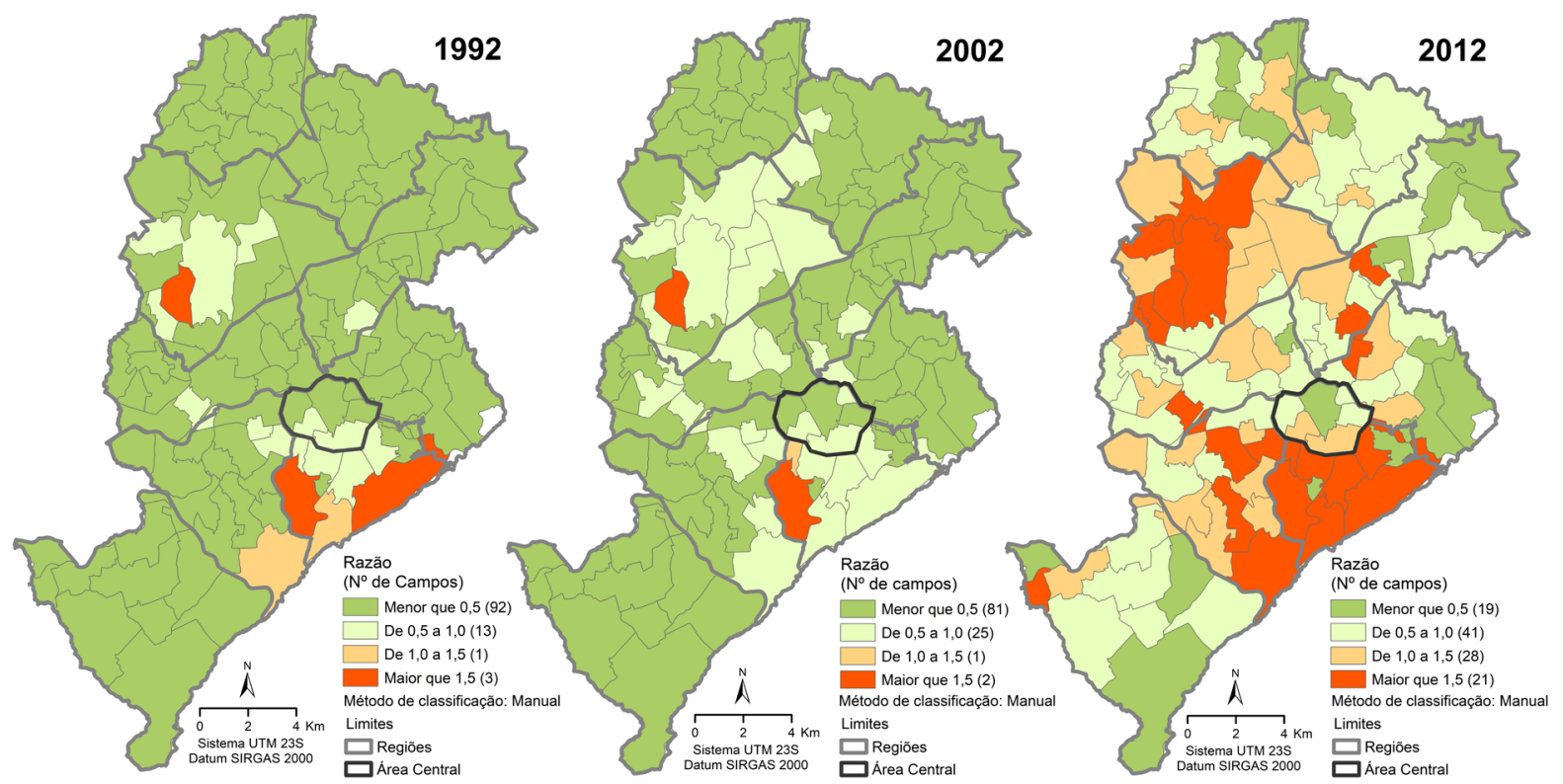

Figura 4. Razão de Mobilidade por Automóvel por Campos em Belo Horizonte, 1992, 2002 e 2012. Fonte: Elaboração própria a partir da base digital das Pesquisas OD (1992, 2002 e 2012) - FJP.

Ainda com base na análise da Figura 4, em 1992, os Campos de maior renda de Belo Horizonte, como Belvedere, Mangabeiras, Santa Lúcia e São Bento, na região CentroSul, além do Campo Castelo, na Regional Pampulha, já registravam um indicador superior a 1, o que significa maior uso do automóvel nas viagens efetuadas. Em 2002, o indicador demonstra redução da proporção de viagens por ônibus em outras áreas do município, como a Pampulha, embora o uso do ônibus tenha registrado aumento em comparação ao automóvel na região Centro-Sul. A hipótese considerada é de que tal situação tem relação com a ampliação do setor de comércio e serviços no eixo sul de Belo Horizonte, atividades intensivas em mão de obra, que tende a se utilizar do transporte coletivo por ônibus. O predomínio do uso do automóvel é observado com maior intensidade na regional Pampulha, Centro Sul e Oeste. É importante enfatizar que a única linha de trem metropolitano em operação em Belo Horizonte não atende os campos da regional Centro-Sul e Pampulha, que apresentaram altos valores para o indicador Razão de Mobilidade por Automóvel. O modo ônibus é majoritário nas periferias do município, especialmente nas regionais Barreiro, Venda Nova, Leste, Norte e Noroeste, que, como anteriormente abordado, concentram menor renda média em Belo Horizonte.

No que tange a distribuição de renda em Belo Horizonte, fator essencial para acesso ao modo de transporte individual, que, a exemplo das demais capitais brasileiras, tem marcada desigualdade socioeconômica. Em geral, observa-se que os Campos com maiores médias se concentram nas regionais Centro-Sul e Pampulha (Figura 6). Em 2000, dos 11 Campos com renda média domiciliar superior $\mathrm{R} \$ 3.000,00$, apenas dois localizavam-se fora do Centro-Sul. Por outro lado, na periferia do município, especialmente aqueles nas regionais Nordeste e Venda Nova, reuniam-se os Campos de menor renda. Ainda que tenha havido um pequeno incremento geral no nível de rendimento médio, o que foi observado para estado de Minas Gerais e Brasil, conforme dados divulgados pelo IBGE, não houve grandes alterações relacionadas à concentração espacial da população de renda mais alta no município. Em suma, as regionais Barreiro, Venda Nova, Norte, Nordeste concentram os estratos com menor rendimento médio, associado a menor utilização do sistema, o que reforça o caráter estrutural da dimensão econômica como fator de acesso ao transporte individual. 


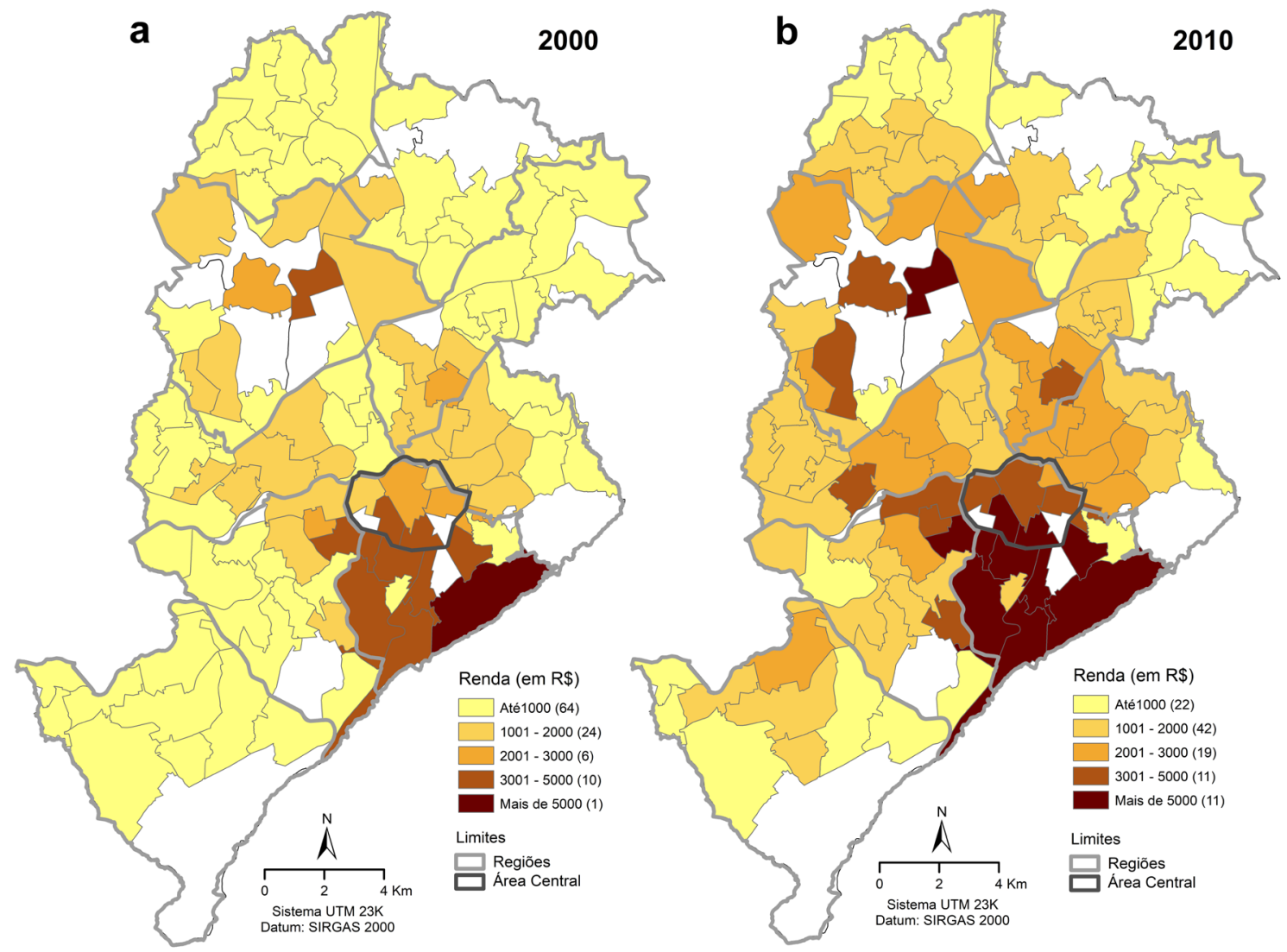

Figura 5. Renda média do responsável pelo domicílio em (em R\$s) por Campos em Belo Horizonte, 2000 e 2010. Fonte: Elaboração própria a partir da base digital das Pesquisas OD (1992, 2002 e 2012) - FJP e Censos Demográficos do IBGE (2000 e 2010)

\section{Projeções de vigens por automóveis: as tendências para 2022}

Considerando o modelo de regressão linear utilizado, cujos valores foram ajustados conforme crescimento da renda média domiciliar (fator de correção), conforme representado na Figura 7, foi possível obervar que o crescimento médio geométrico de 2,37\% a.a. nas viagens por automóvel em Belo Horizonte nos anos de 2012 para 2022 (variação total de 387.411 viagens). A análise das projeções revela a continuidade da tendência de expansão das viagens por automóvel, porém, de maneira heterogênea no território municipal (Figura 7a). O perímetro da Avenida do Contorno, (que limita a área originalmente planejada da cidade), é aquela com continua com maior número de deslocamentos por automóvel, de acordo com as projeções. Campos como Belvedere, Sion, Savassi, São Bento, Santa Lucia e Lourdes, todos na regional Centro-Sul, e o Campo Cidade Universitária, na Regional Pampulha, apresentaram o maior número de viagens por automóveis. São áreas habitadas por população de maior renda ou, ainda, locais que concentram equipamento de interesse público. Os menores valores totais de deslocamento por automóvel foram observados nas periferias das regionais Norte, Nordeste, Leste e Venda Nova.

Em relação à taxa de crescimento das viagens (Figura 7b), os maiores percentuais de crescimento foram observados nas periferias, principalmente as localizadas nas regionais Barreiro, Noroeste, Norte e Venda Nova, que acusaram taxas superiores a 3\% entre o ano de 2012 e 2022. Esses resultados se relacionam ao aumento da renda, inclisive nas áreas periféricas do município, o que sugere que muitas famílias de menor poder aquisitivo obtiveram e passaram a utilizar o automóvel, entendido não apenas como meio de locomoção, mas como símbolo de inserção no mercado de consumo. Foi essa 
a solução individual encontrada para as precárias condições do transporte público em Belo Horizonte, principalmente no que se refere aos tempos de viagem e opções de trajeto para os moradores de áreas mais distantes dos espaços de consumo. Por sua vez, embora concentre o maior volume de viagens, na Regional Centro-Sul se observou os menores índices de crescimento dos deslocamentos por automóvel, o que sugere que o uso do automóvel permaneceu constante com os níveis dos anos anteriores.
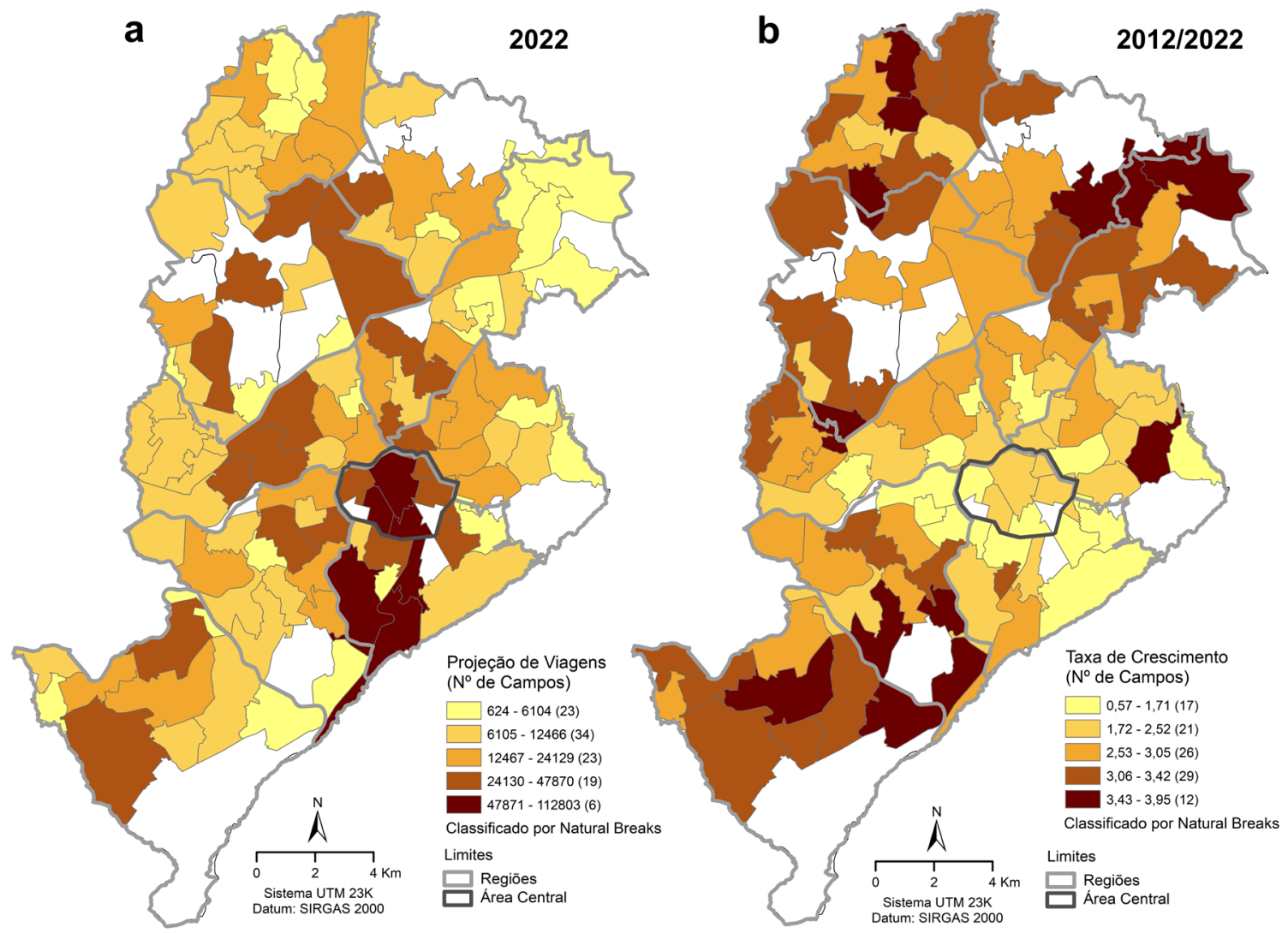

Figura 6. Projeções ajustadas do número (a) e da taxa de crescimento (b) das viagens por automóvel em Belo Horizonte, 2022. Fonte: Elaboração própria a partir da base digital das Pesquisas OD (1992, 2002 e 2012) - FJP e Censo Demográfico IBGE 2000 e 2010

É importante salientar que o modelo não leva em conta possíveis intervenções relacionadas ao transporte público posteriores ao ano de 2012, data da última Pesquisa Origem e Destino/RMBH. Porém, ações importantes previstas pelo Plano Diretor de Mobilidade Urbana de Belo Horizonte (PlamMob-BH), como a ampliação das intervenções com vistas a priorizar o transporte coletivo, foram observadas após a OD 2012. Dentre tais intervenções, merece destaque a implantação do sistema Bus Rapid Transport (BRT) nos corredores viários das avenidas Vilarinho, Dom Pedro I, Presidente Antonio Carlos e Cristiano Machado, no ano de 2014 como parte das ações de mobilidade para o evento Copa do Mundo 2014, a qual Belo Horizonte foi uma das sedes.

\section{Considerações finais}

O crescimento do transporte individual em detrimento do transporte público é um dos principais desafios das políticas de mobilidade urbana das grandes cidades brasileiras. Belo Horizonte não foge a essa condição. Como descrito nesse trabalho, verificou-se 
nos últimos trinta anos substancial crescimento da frota de automóveis. Como consequência, o município experimentou considerável elevação dos percentuais de participação desse modo de transporte. O resultado é a manutenção de um círculo vicioso, de maneira que um maior número de viagens por transporte particular resulta em aumento dos congestionamentos, dos tempos gastos, dos níveis de poluição e a deterioração da qualidade do transporte público (deslocamentos mais demorados, maior tempo de espera, veículos com maior lotação).

Em geral, a solução adotada individualmente tem sido a aquisição de um veículo particular, especialmente o automóvel e a motocicleta. Contudo, é importante salientar algumas mudanças nas práticas atuais de transportes, entre elas a tendência característica das novas gerações que demonstram pouco interesse na aquisição de veículo particular, mas que o utilizam ocasionalmente a partir de aplicativos. Esse novo fenômeno tem dado espaço à novas concepções como o MaaS - Mobility as a Service - discutidas no trabalho de Jittrapirom et al. (2017). Nota-se que as análises desenvolvidas neste trabalho não consideraram esse serviço, o que gera possibilidades para futuras linhas de pesquisa

A análise das viagens por ônibus e automóveis nos anos de 1992, 2002 e 2012, tendo como origem os Campos de Belo Horizonte, revela a elevada concentração dos fluxos no centro do município. Isso ocorre pela maior demanda produzida pelo número de estabelecimentos de comércio e serviços nessa região. Porém, é possível verificar a tendência de desconcentração das atividades econômicas no município desde 1992, com a maior participação de centralidades na periferia, como Barreiro e Venda Nova, além de bairros do chamado Vetor Norte. Verifica-se, ainda, o aumento de viagens no eixo sul de Belo Horizonte, justificado pelo espraiamento das atividades comerciais em direção a bairros da regional Centro-Sul, como Sion e Belvedere. A gradativa desconcentração das atividades do centro de Belo Horizonte é um importante fator a ser considerado nas políticas de mobilidade urbana, já que pode reduzir a saturação de alguns corredores de tráfego, além de possibilitar a redução das distâncias percorridas e dos tempos de viagem.

As projeções para 2022, apesar de indicam a permanência da concentração das viagens por automóvel na área central e pericentral, um expressivo crescimento do transporte individual nas periferias urbanas de Belo Horizonte, dado o crescimento relativo da renda entre os anos de 2000 e 2010 e a baixa eficácia do transporte público. Nesse sentido, tais regiões exigem maiores intervenções voltadas para o transporte coletivo, como a ampliação do transporte ferroviário, tendo em vista que as regiões Barreiro e Centro-Sul, áreas com expressivo número e crescimento da participação do transporte por automóvel, não são atualmente atendidas pela linha de trem urbano existente.

A necessidade de melhorias no transporte público torna-se a principal medida de intervenção frente à realidade que se apresenta para os próximos anos em Belo Horizonte. O Plano de Mobilidade Urbana de Belo Horizonte (Planmob), desenvolvido entre 2007 e 2010, elenca entre seus objetivos estratégicos "tornar o transporte público mais atrativo frente ao transporte individual”. A implantação do sistema BRT em 2014 faz parte das ações do plano para estimular o uso do transporte público (BHTRANS, 2012). Espera-se que a próxima Pesquisa OD/RMBH, a ser realizada em 2022, torne possível avaliar os efeitos da implantação do sistema BRT sobre o número de viagens por ônibus e automóveis em Belo Horizonte, bem como as demais ações planejadas pelo Planmob. 


\section{Q Bibliografia}

» ANTP. (1997). Transporte Humano: cidades com qualidade de vida. São Paulo, Associação Nacional de Transportes Públicos, 312 p.

» Araújo, M. R. M., Oliveira, J. M., Jesus, M. S., S. Á, N. R., Santos, P. A. C., e Lima, T. C. (2011). Transporte público coletivo: discutindo acessibilidade, mobilidade e qualidade de vida. Psicologia \& Sociedade 23, (3), 574-582.

" Barbosa, J. L. (2014). A mobilidade urbana como expressão do Direito à Metrópole. In: Limonad, E. e Castro, E. R. (Org.). Um novo planejamento para um novo Brasil? Rio de Janeiro, Letra Capital, p. 187-201.

» Berman, M. (2007). Tudo o que é sólido desmancha no ar: a aventura da modernidade. São Paulo: Companhia das Letras.

»BHTRANS. (2012). Plano diretor de mobilidade urbana de Belo Horizonte PlamMob-BH - Relatório final. Belo Horizonte: Prefeitura de Belo Horizonte. Disponível em: <http://www.bhtrans.pbh.gov.br/portal/pls/portal/!PORTAL. wwpob_page.show?_docname=9604263.PDF, acesso em 14 de maio de 2017.

"Cardoso, L. (2007). Transporte público, acessibilidade urbana e desigualdades socioespaciais na Região Metropolitana de Belo Horizonte. Belo Horizonte, Tese de doutorado em Geografia, Universidade Federal de Minas Gerais.

"Carvalho, C. H. R. (2016). Desafios da mobilidade urbana no Brasil. Texto para Discussão (2198). Brasília, Instituto de Pesquisa Aplicada (IPEA). Disponível em: <http://repositorio.ipea.gov.br/bitstream/11058/6664/1/td_2198.pdf>. Acesso em: 20 maio 2017.

»Cheng, Y. H., e Chen, S. Y. (2015). Perceived accessibility, mobility, and connectivity of public transportation systems. Transportation Research Part A 77, 386-403

»Costa, P. B., Neto, G. C. M. e Bertolde, A. I. (2017). Urban mobility indexes: a brief review of the literature. Transportation Research Procedia ${ }_{25}$ C, 3649-3659.

» DEAR. M. J., SCOTT, A. J. (1981). Urbanization and urban planning in capitalist societies. London: Methuen.

"Ewing, R., e Handy, S. (2009). Measuring the Unmeasurable: Urban Design Qualities Related to Walkability. Journal of Urban Design 14 (1), 65-84. doi:10.1080/13574800802451155.

» Fundação João Pinheiro. (2017). Nova Lima. Disponível em: <http://acervo. ci.fdc.org.br/AcervoDigital/Cadernos\%2ode\%2old\%C3\%A9ias/2009/0910. pdf $>$. Acesso em: 25 março 2017.

" Gomide, A. A. (2006). Mobilidade urbana, iniquidade e políticas sociais. Políticas sociais acompanhamento e análise 12, 242-250.

"Gutiérrez, A. (2012). ¿Qué es la movilidad? Elementos para (re)construir las definiciones básicas del campo del transporte. Bitacora 21, (2), 61-74. doi: 10.15446 / bitacora

" Harris, C. D. (1954). The market as a factor in the localization of industry in the United States. Annals of the Association of American Geographers 44, (4), 315-348. doi:10.1080/00045605409352140. 
» IBGE. (2017). Estimativas populacionais dos municípios em 2017. Disponível em: https://cidades.ibge.gov.br/v4, acesso em os de Setembro de 2017.

» Jayme, J. G., Trevisan, E. (2012). Intervenções urbanas, usos e ocupações de espaços na região central de Belo Horizonte. Civitas 12 (2), 359-377.

» Jittrapirom, P., Caiati, V. Feneri, A-M., Ebrahimigharehbaghi, S., AlonsoGonzález, M. J., e Narayan, J. (2017). Mobility as a Service: A Critical Review of Definitions, Assessments of Schemes, and Key Challenges. Urban Planning 2, (2), 13-35. doi: 10.17645/up.v2i2.931.

» Jones, S.R. (1981). Accessibility measures: a literature review. TRRL Report 967, Transport and Road Research Laboratory. Crowthorne, Berkshire.

»Lefébvre, H. (1969). O direito à cidade. São Paulo: Documentos.

" Litman, T. (2017). Evaluating Transportation Land Use Impacts: Considering the Impacts, Benefits and Costs of Different Land Use Development Patterns - Report. Victoria: Victoria Transport Policy Institute (VTPI), Canadá. Disponível em: <http://www.vtpi.org/landuse.pdf>. Acesso em: 30 maio 2017.

"Lobo, C., Cardoso, L., e Magalhães, D. (2013). Acessibilidade e mobilidade espaciais da população na Região Metropolitana de Belo Horizonte: análise com base no censo demográfico de 2010. Caderno Metropolitano 15, (30), 513-533.

»Lobo, C., Cardoso, L., e Matos, R. E. S. (2012). Transporte público coletivo por ônibus em Belo Horizonte: a eficiência de acessibilidade com base na pesquisa domiciliar origem e destino de 2002. Revista dos Transportes Públicos (ANTP) 34.

» Maggi, E., e Vallino, E. (2016). Understanding urban mobility and the impact of public policies: The role of the agent-based models. Research in Transportation Economics 55, 50-59.

》 Minas Gerais. (2002). Relatório consolidado pesquisa origem e destino 2001-2002. Fundação João Pinheiro.

" Minas Gerais. (2012). Pesquisa Origem e Destino 2012 - Base de dados. Agência de Desenvolvimento da Região Metropolitana de Belo Horizonte.

" Mitra, S. K., e Saphores, J.D. M. (2016). The value of transportation accessibility in a least developed country city - The case of Rajshahi City, Bangladesh. Transportation Research Part A 89, 184-200.

»Neto, O. L. (2004). Um novo quadro institucional para os transportes públicos: condição sine qua non para a melhoria da mobilidade e acessibilidade metropolitana. In Santos, E.; Aragão, J., (Orgs.). Transporte em tempos de reforma - estudo sobre o transporte urbano. Editora Universitária da UFRN, EDUFRN, Natal, p. 193-216.

» Nicolas, J.P., Pochet, P., e Poimboeuf, H. (2003). Towards sustainable mobility indicators: application to the Lyons conurbation. Transport Policy 10 (3), 197-208.

"Oliveira, G. M., e Silva, A. N. R. (2015). Desafios e perspectivas para avaliação e melhoria da mobilidade urbana sustentável: um estudo comparativo de municípios brasileiros. Transportes 23 (1), 59-68.

»Pyrialakou, V. D., Gkritza, K., e Fricker, J. D. (2016). Accessibility, mobility, and realized travel behavior: Assessing transport disadvantage from a policy perspective. Journal of Transport Geography 51, 252-269. doi:10.1016/j. jtrange0.2016.02.001.

"Raia Junior, A. A. (2000). Acessibilidade e mobilidade na estimativa de um índice de potencial de viagens utilizando redes neurais artificiais e sistemas de 
informação. São Carlos, Tese de doutorado em Engenharia Civil Transportes, Escola de Engenharia de São Carlos, Universidade de São Paulo.

» Resende, P. T. V., e Souza, P. R. (2009). Mobilidade urbana nas grandes cidades brasileiras: Um estudo sobre os impactos do congestionamento. Caderno de Ideias - Clog10.

» Rodrigue, J. P., Comtois, C., e Slack, B. (2013). The Geography of Transport Systems ( $3^{\mathrm{a}}$ ed). Routledge, London.

» Schoenau, M., e Müller, M. (2017). What affects our urban travel behavior? A GPS-based evaluation of internal and external determinants of sustainable mobility in Stuttgart (Germany). Transportation Research Part F 48, 61-73.

"Sismob-BH. (2015). Sistema de Informações de Acessibilidade e Sustentabilidade de Belo Horizonte. Indicadores Populacionais. Disponível em: <http://www. bhtrans.pbh.gov.br/portal/page/portal/portalpubicodl/Temas/Observatorio/ SISMOBBH-2013/item8_10_SisMobBH_DadosAbertos_2017_01_23.pdf>. Acesso em: 04 junho 2017.

" Tagore, M. R., e Sikdar, P. K. (1995). A new accessibility measure accounting mobility parameters. In: World Conference on Transport Research 7, Sydney. Proceedings... Sydney, Australia: WCTR.

»United Nations. (2014). World Urbanization Prospects - The 2014 Revision - Final Report. Department of Economic and Social Affairs, Population Division (ST/ ESA/SER.A/366). Disponível em: <https://esa.un.org/unpd/wup/Publications/ Files/WUP2014-Report.pdf>. Acesso em: 25 maio 2017.

»Vasconcellos, E. A. (2000). Transporte urbano nos países em desenvolvimento: reflexões e propostas 3. São Paulo: Annablume.

»Vasconcellos, E. A. (2001). Transporte urbano, espaço e equidade - Análise das políticas públicas 2. São Paulo: Annablume.

»Vasconcellos, E. A., Carvalho, C. H. R., Pereira, R. H. M. (2011). Transporte e mobilidade urbana. Brasília. CEPALIIPEA.

"Vulevic, A. (2016) Accessibility concepts and indicators in transportation strategic planning issues: theoretical framework and literature review. Logistics \& Sustainable Transport 7 (1), 58-67. doi:10.1515/jlst-2016-00o6.

»Waldvogel, B. (1998). Técnicas de projeção populacional para o planejamento regional. Belo Horizonte, CEDEPLAR.

» Zandonade, P., Moretti, R. (2012). O padrão de mobilidade de São Paulo e o pressuposto de desigualdade. EURE 38, 77-97.

\section{Daniela Antunes Lessa / dani.antunes@gmail.com}

Engenheira Civil (Universidade Federal de Minas Gerais), Mestre em Transportes (Universidade Federal de Minas Gerais/UFMG) e Doutoranda em Organização do Espaço/Geografia (Universidade Federal de Minas Gerais/UFMG).

\section{Giovanni Candido Miranda / giovannicmiranda@yahoo.com.br}


Carlos Lobo / carlosfflobo@gmail.com

Geógrafo (Universidade Federal de Minas Gerais) e Doutor em Geografia (Universidade Federal de Minas Gerais). Subcoordenador e Professor do Programa de Pós-Graduação em Geografia do Instituto de Geociências da Universidade Federal de Minas Gerais.

\section{Leandro Cardoso / leandro@etg.ufmg.br}

Geógrafo (Universidade Federal de Minas Gerais), Mestre em Geografia (Universidade Federal de Minas Gerais) e Doutor em Geografia (Universidade Federal de Minas Gerais). Professor do Departamento de Engenharia de Transportes e Geotecnia da Universidade Federal de Minas Gerais. 